\title{
Experimental characterisation of rate-dependent compression behaviour of fibre reinforced composites
}

\author{
Mehtab V. Pathan ${ }^{1, *}$, Borja Erice ${ }^{1}$, Sathiskumar A. Ponnusami ${ }^{1}$, and Nik Petrinic ${ }^{1}$ \\ ${ }^{1}$ Department of Engineering Science, University of Oxford, Parks Road, Oxford, OX1 3PJ, United Kingdom
}

\begin{abstract}
Fibre reinforced polymers (FRP) materials are being increasingly used for aerospace and automotive structural applications. One of the critical loading conditions for such applications is impact, consequently, understanding of the composite behavior under such loads becomes critical for structural design. The analysis and design process for achieving impact-resistant composite structures requires rate-dependent constitutive models, which, in turn, requires material properties of the composite over a range of strain rates. It is, therefore, the objective of the research to investigate the strain rate-dependent behavior of fiber reinforced composites under compressive loads for a wide range of fiber orientations. Quasi-static $\left(\approx 1 \mathrm{e}-3 \mathrm{~s}^{-1}\right)$ and high loading $\left(\approx 200 \mathrm{~s}^{-1}\right)$ rates are considered for the experimental study. Accordingly, two different test setups are utilized, a screw-driven universal testing machine for quasi-static tests and a Split Hopkinson Pressure Bar (SHPB) system for dynamic tests. The stress-strain response of the composite is reported for the different fiber orientations and the strain rates, revealing the rate-dependent characteristics of the carbon fiber reinforced composite. From the test results, it is observed that, the dependency of the fracture strength on the loading rate is significant. The results are summarised in terms of the failure envelope in the transverse compression-in-plane shear $\sigma_{22}-\sigma_{12}$ plane for the two strain rates.
\end{abstract}

\section{Introduction}

Fibre composites are now a popular choice of material in the aerospace industry given their superior specific strength and stiffness properties. Due to the viscoelastic nature of polymeric matrix material, mechanical response of FRPs under high strain rates is markedly different to that measured under quasi-static loading conditions. Thus, proper characterization of the strain rate dependency of FRPs is critical to allow for the optimal design of composite structures under dynamic loading.

There have been few studies dealing with experimental characterisation of the strain rate dependent behaviour of uni-directional(UD) FRPs under multi-axial load states [1-4]. Koerber et al. [1] studied the in-plane compressive response of UD carbon fibre composites using off-axis coupons at quasi-static (QS) and high rate (HR). An average increase of $12 \%$ and $45 \%$ was observed for the case of transverse compressive modulus and failure strength with strain rate, respectively. However, no significant change in the ultimate failure strain was found. Naresh et al. [2] studied the tensile response of GFRP, CFRP and hybrid composites at HR using a droptower setup. No significant strain rate dependency was observed for the case of tensile strength and modulus of CFRP material. More recently, Tao et al. [3] studied the QS and HR inter-fibre failure behaviour of off-axis UD GFRP coupons under transverse tension and compression. Significant expansion of

\footnotetext{
*e-mail: mehtab.pathan @eng.ox.ac.uk
}

the $\sigma_{22}-\tau_{12}$ failure envelope was observed with increasing strain rate.

Despite the above mentioned studies, test data of FRPs subjected to multi-axial load states at QS and HR remains scarce, which limits validation and development of new strain rate dependent failure theories. The present study aims to characterize the transverse compression-in plane shear behaviour of CFRP coupons under QS $\left(\approx 1 \mathrm{e}-3 \mathrm{~s}^{-1}\right)$ and dynamic loading rates $\left(\approx 200 \mathrm{~s}^{-1}\right)$. The paper outline is as follows: details of material preparation, specimen design and experimental setups are given in section 2 , section 3 presents the experimental results followed by discussion and conclusion in section 4 and section 5 , respectively.

\section{Experimental details}

\subsection{Material and specimen preparation}

A material system consisting of carbon fibre and highlytoughened matrix (belonging to same family of resins as Hexcel 8552) was used for this study. UD CFRP panels of $5 \mathrm{~mm}$ nominal thickness were manufactured using autoclave curing process. Traditionally, a block specimen design [1,2] is employed for compression testing due to its simplicity and ease of specimen preparation. However, based on previous experience it was found that the block design showed a strong tendency to fail near the specimen-loading fixture interface due to presence of high lateral forces due to friction, despite using appropriate 
lubrication measures. Thus, a waisted specimen design as shown in (fig. 1) was used in this study. Following $[1,3,4]$, off-axis coupons were used to induce a combined compression-shear load state in the material. Initially, rectangular blanks of size were cut using water-jet in the $30^{\circ}, 45^{\circ}, 60^{\circ}$ and $90^{\circ}$ orientation with respect to the fibre direction. Coupons were subsequently ground to required dimensions using a series of grinding wheels, resulting in high planarity of specimen surfaces with a smooth surface (roughness $<3$ micron), thus limiting premature failure of coupons in the presence of manufacturing defects. At least three specimens were tested for a given configuration to ensure repeatability of the experimental results.

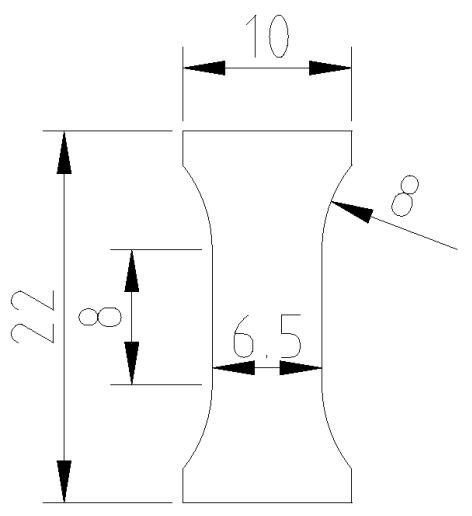

Fig. 1. Geometric dimensions of compression specimens.

\subsection{Experimental setup for quasi-static tests}

Quasi-static compression tests were performed using a Zwick/Roell screw-driven universal testing machine. The applied load was recorded using a $100 \mathrm{kN}$ resistive load cell whilst Digital Image Correlation (DIC) was used to obtain specimen strains. The specimen was loaded in compression under displacement control mode with a crosshead velocity of $0.48 \mathrm{~mm} / \mathrm{min}$, resulting in a strain rate $\dot{\epsilon}=10 \mathrm{e}-3 \mathrm{~s}^{-1}$. A custom designed loading frame consisting of two ball bearings was used to load the specimen in order to minimize the effect of any eccentricity loading arising from non-planarity in the specimen surfaces. The loading fixture was lubricated with a thin layer of WD40 solution to avoid any barrelling effect of specimen due to friction. A uEye 2 SE camera was used to capture images with a frame rate of $1 \mathrm{fps}$. At least 3 specimen were tested for a given off-axis configuration and loading rate.

\subsection{Experimental setup for high rate tests}

A classical Split-Hopkinson Pressure Bar (SHPB) system was used for high rate compression tests, schematic of which is shown in fig. 2. For sake of brevity, theoretical background and data reduction techniques of SHPB test is not included in this paper, readers are referred to texts by Kolsky [5] and Gray [6] for full details.
Cylindrical, solid bars made of Ti6Al4V with $\mathrm{E}=110 \mathrm{GPa}, \rho=4400 \mathrm{~kg} / \mathrm{m}^{3}$ and diameter $=16 \mathrm{~mm}$ were used in the SHPB setup. The lengths of the striker, input and output bars were 2700, 2510 and $2510 \mathrm{~mm}$, respectively. A Kirana ultra-high speed camera was used to capture images with a frame rate of 500,000 fps and image resolution of $924 \times 768$ pixels $^{2}$ using flash light illumination. Three sets of strain gauges were used to measure the bar signals (see fig. 2), two in the input bar located at a distance of $2250 \mathrm{~mm}$ and $430 \mathrm{~mm}$ and one in the output bar at a distance of $229 \mathrm{~mm}$ from the specimen-bar interface, respectively.

Results obtained from SHPB are only valid under the assumptions of linear wave propagation in the bar, presence of dynamic equilibrium in the specimen and constant strain-rate in the specimen. The first assumption is satisfied due to the large aspect ratios of the bars used in the experimental setup and appropriate selection of bar material. The dynamic force equilibrium requirement which refers to load balance between the input bar- and output barspecimen interfaces ensures that the measured dynamic properties are representative of the material and are not influenced by inertial loading. The forces at the input and output bars were analysed using the measured bar signals and were found to be effectively same throughout the test period. All SHPB tests were performed using a $3 \mathrm{~mm} \mathrm{Neo-}$ prene pulse shaper to facilitate a constant strain rate in the specimen throughout the test.

\section{Experimental Results}

\subsection{Quasi-static strain rate results}

All subsequent results are presented in a normalized format, where all the specimen axial stresses are normalised by the quasi-static, ultimate transverse compressive strength of the IM7-8552 Hexcel material system given in Table 2 of Koerber et al. [1].

Results for different off-axis coupons under quasistatic loading performed at a strain rate of $\epsilon_{\dot{Q} S}=1 \mathrm{e}-3 \mathrm{~s}^{-1}$ are given in figs. 3 to 6 . Each line in the plots refer to a different test specimen. The test results are largely repeatable with failures consistently occurring in the gauge region. This can be attributed to the improved specimen design and preparation methods discussed in section 2.1.

In terms of stress-strain curves, the response of transverse $90^{\circ}$ coupon (see fig. 3) is mostly linear while other off-axis coupons (figs. 4 to 6 ) show a large degree of nonlinearity under compression loading. It is to be noted that significant fibre rotation was observed for the case of $30^{\circ}$ and $45^{\circ}$ specimen.

Results of each off-axis configuration under QS loading are summarized in fig. 7. As expected, the $30^{\circ}$ specimen was the strongest followed by $60^{\circ}, 45^{\circ}$, and $90^{\circ}$ all of which had similar ultimate failure stresses. $45^{\circ}$ specimen on an average exhibited the largest axial strain to failure of $8 \%$ followed by $60^{\circ}, 30^{\circ}$ and $90^{\circ}$ coupons.

The failure patterns of each off-axis configuration is shown in fig. 7 . The $30^{\circ}$ specimen failed under in-plane 


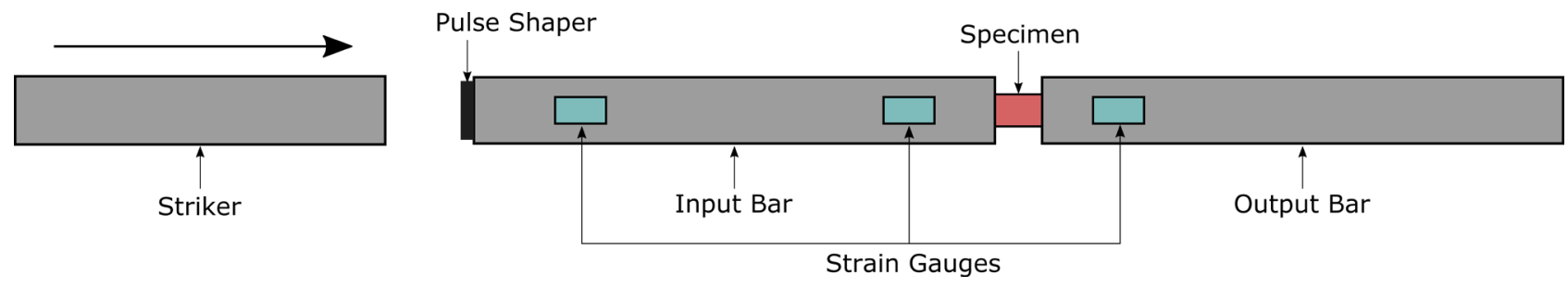

Fig. 2. Schematic of the SHPB setup.

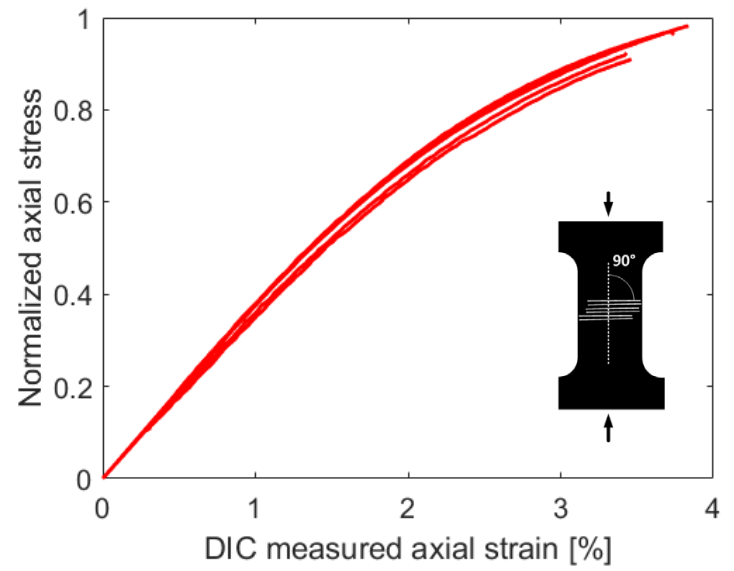

Fig. 3. Stress-strain response of $90^{\circ}$ coupons under quasi-static compression.

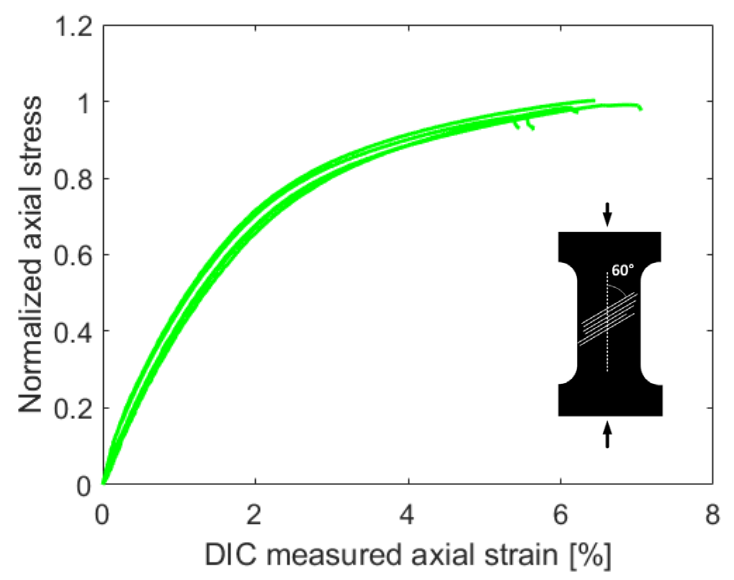

Fig. 4. Stress-strain response of $60^{\circ}$ coupons under quasi-static compression.

shear mode characterized by a clean crack oriented along the fibre direction. While, $45^{\circ} 60^{\circ}$ and $90^{\circ}$ failed under a compression dominated load state characterized by an inclined failure surface in the through-thickness direction. The failure patterns of all specimens are in good agreement to those observed by Koerber et al [1].

\subsection{High strain rate results}

The response of the off-axis coupons under high-rate loading is as given in figs. 9 to 12 . The average strain rates in

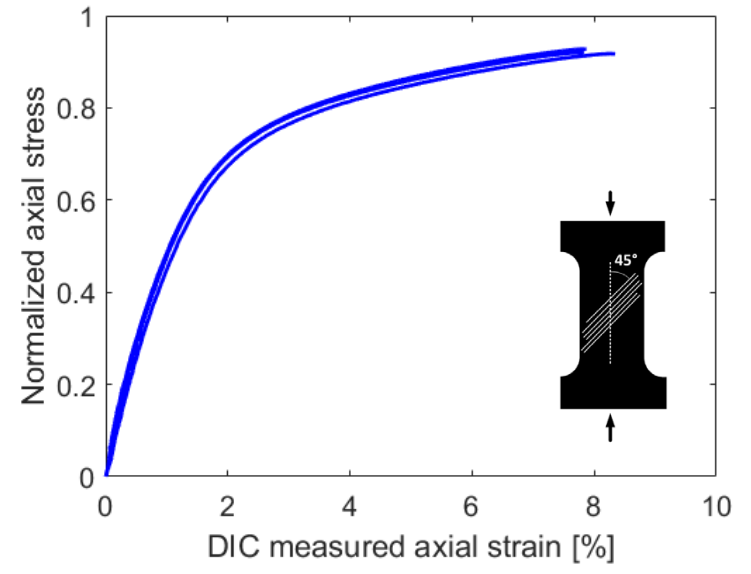

Fig. 5. Stress-strain response of $45^{\circ}$ coupons under quasi-static compression.

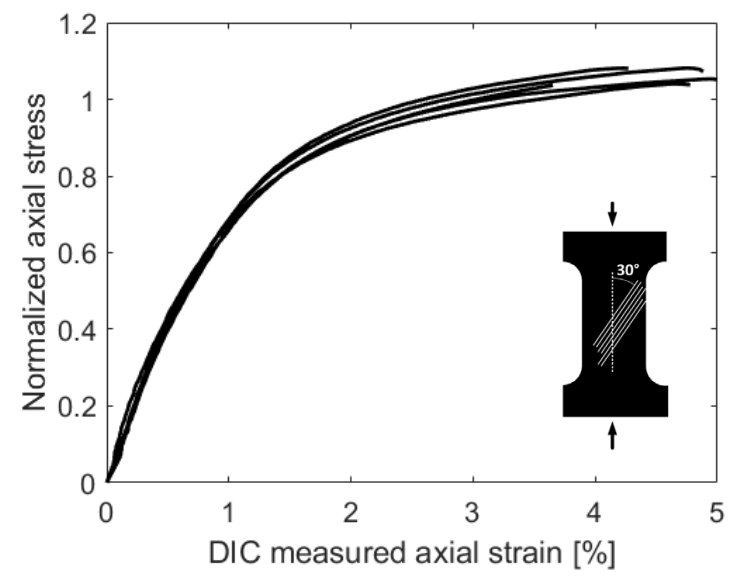

Fig. 6. Stress-strain response of $30^{\circ}$ coupons under quasi-static compression.

the $30^{\circ}, 45^{\circ}, 60^{\circ}$ and $90^{\circ}$ specimen were $222 \mathrm{~s}^{-1}, 256 \mathrm{~s}^{-1}$, $200 \mathrm{~s}^{-1}$ and $182 \mathrm{~s}^{-1}$, respectively.

\section{Discussion}

Comparison of each off-axis configuration under different strain rates is as given in fig. 13. The effect of strain rate on the material response is clearly pronounced leading to an overall increase in failure strength and decrease in failure strain. However, interestingly the transverse $90^{\circ}$ coupon exhibits an increase of $30 \%$ for the failure strain for HR as compared to the QS loading rate. 


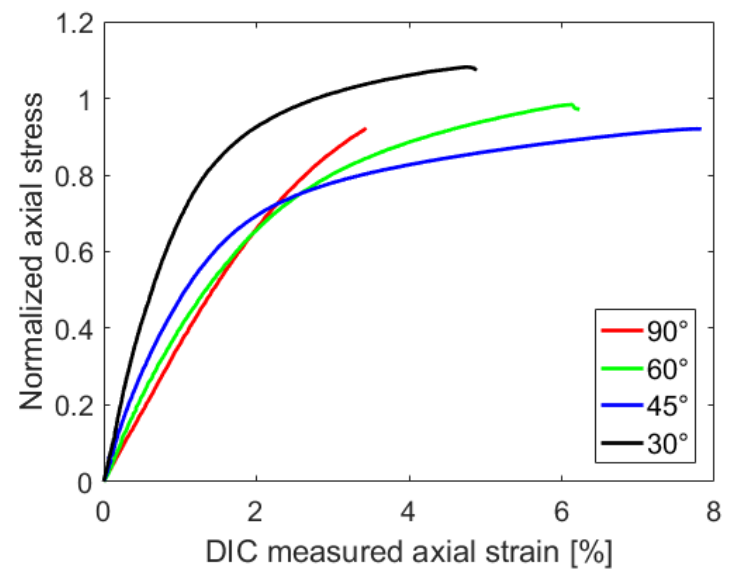

Fig. 7. Summary of stress-strain response of off-axis coupons under quasi-static compression.

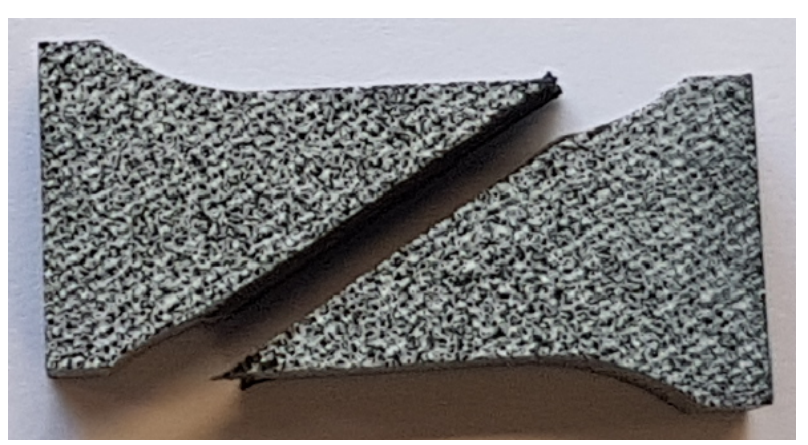

(a) $30^{\circ}$

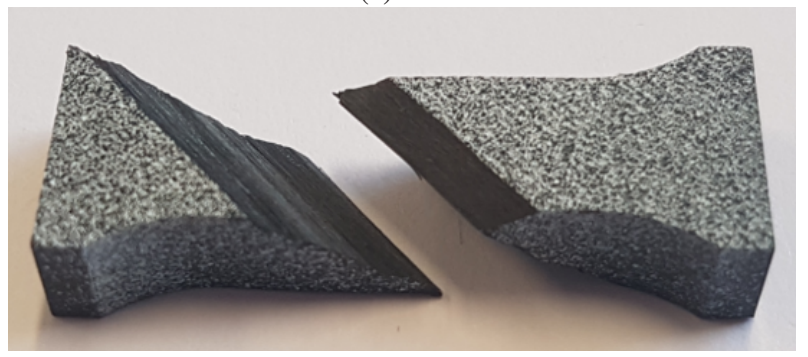

(b) $45^{\circ}$

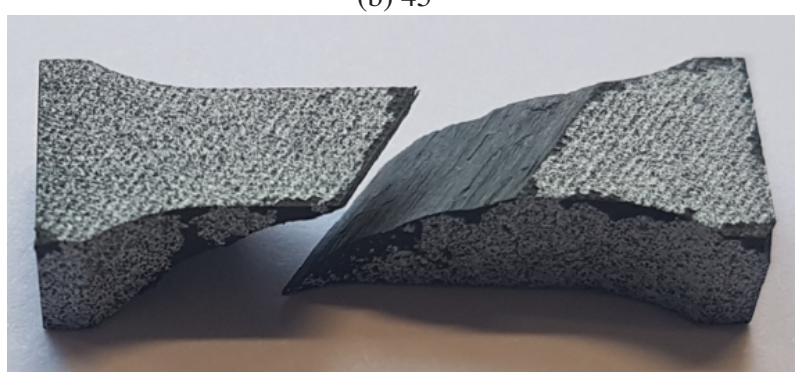

(c) $60^{\circ}$

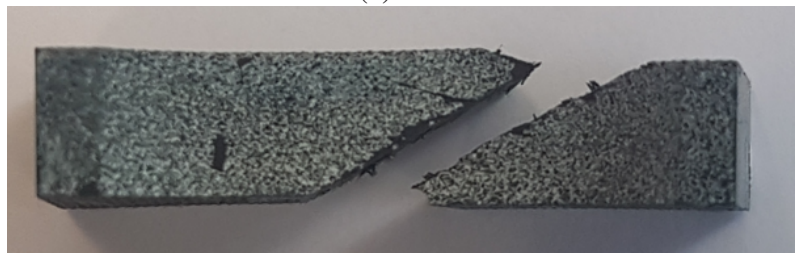

(d) $90^{\circ}$

Fig. 8. Observed failure patterns for different off-axis specimen under quasi-static compression.

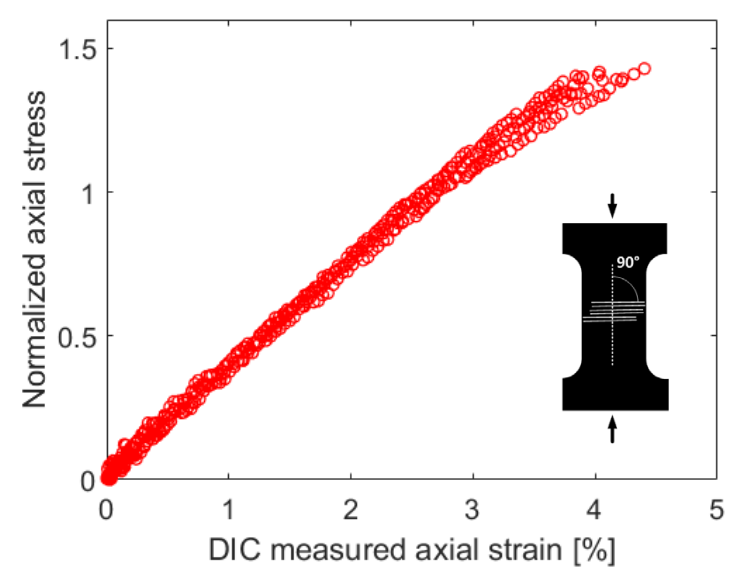

Fig. 9. Stress-strain response of $90^{\circ}$ coupons under dynamic compression.

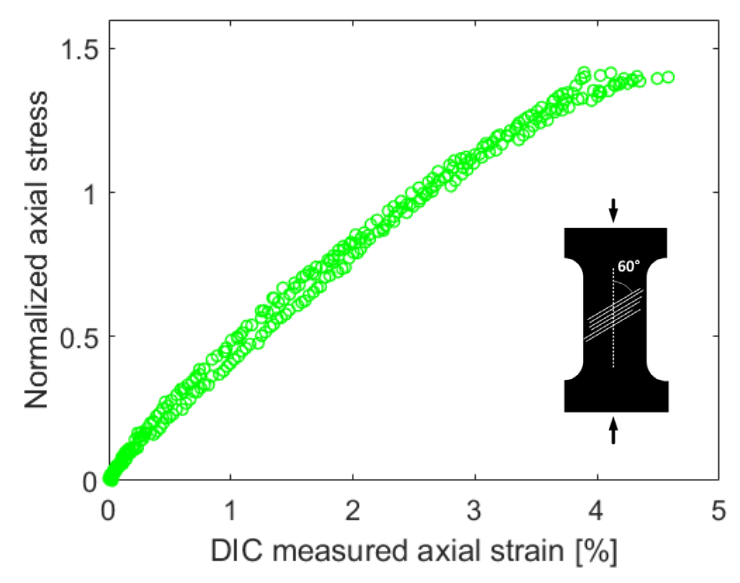

Fig. 10. Stress-strain response of $60^{\circ}$ coupons under dynamic compression.

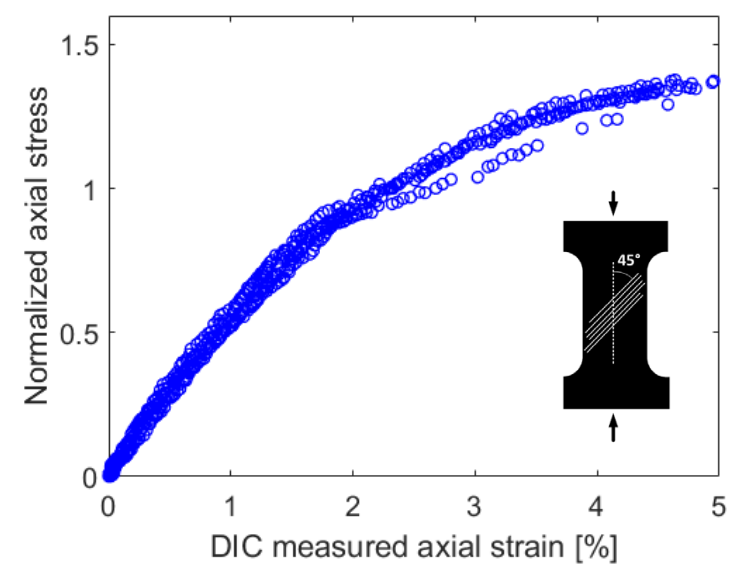

Fig. 11. Stress-strain response of $45^{\circ}$ coupons under dynamic compression. 


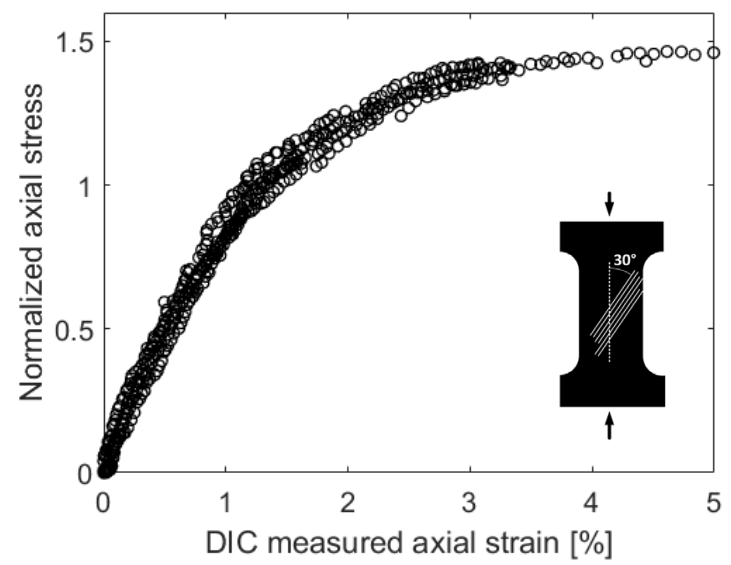

Fig. 12. Stress-strain response of $30^{\circ}$ coupons under dynamic compression.

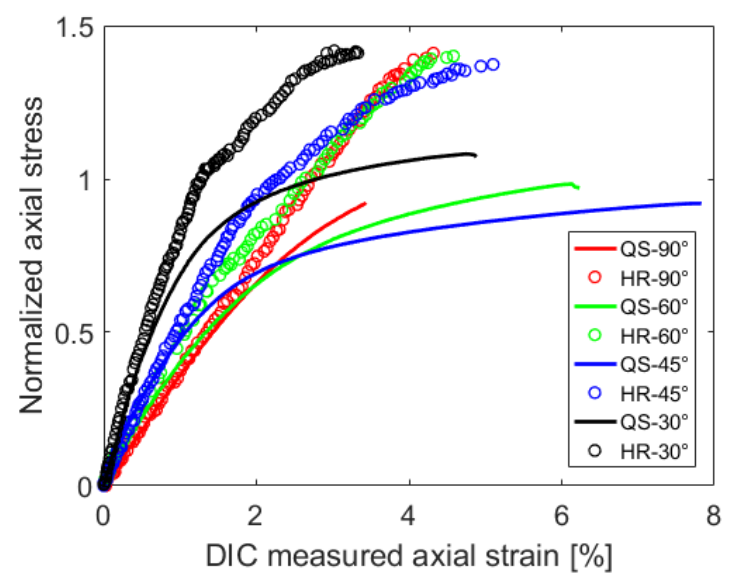

Fig. 13. Comparison of the response of off-axis coupons under quasi-static and dynamic compression.

Results for the measured axial modulus and ultimate strength for QS and HR are summarized in table 1. For case of axial moduli, an increase of $13.5 \%$ is observed for the case of $30^{\circ}$ coupon at high rate, while other axial moduli are scarcely affected in comparison. For the case of ultimate strength, a large, average increase of $43 \%$ is seen with increase in strain rate.

It is informative to construct a material failure envelope under quasi-static and dynamic conditions by transforming the axial coupon stress in the material coordinate system using the following relations:

$$
\begin{aligned}
\sigma_{22} & =\sigma_{x x} \sin ^{2} \beta \\
\tau_{12} & =-\sigma_{x x} \sin \beta \cos \beta
\end{aligned}
$$

Puck failure theory [7] is widely accepted to give good predictions of the inter-fibre failure in the $\sigma_{22}-\sigma_{12}$ plane and is included in fig. 14 for comparison. It is to be noted that due to lack of the longitudinal shear strength $\left(S_{L}\right)$ data, values of $S_{L}=90$ and $S_{L}=135 \mathrm{MPa}$ were used to generate the QS and HR Puck theoretical predictions, re-

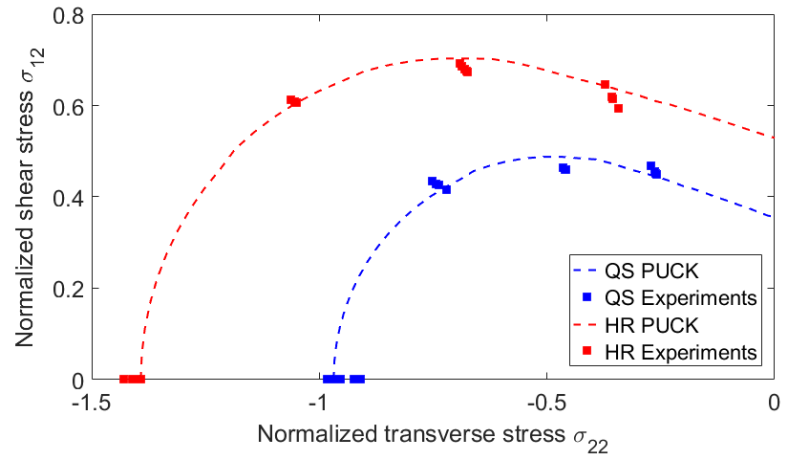

Fig. 14. Experimental and theoretical failure envelopes in $\sigma_{22}-$ $\sigma_{12}$ plane.

spectively. Similar values have been reported for a toughened matrix composite in [1]. The significant expansion of the failure envelope with increasing strain rate is clearly evident from fig. 14, further highlighting the importance of analysing the material strain rate dependency during component design. Puck failure theory provides reasonable predictions of the quasi-static and high rate experimental failure envelopes for the given material but requires further assessment regarding the longitudinal shear strength and its performance in the tensile transverse stress quadrant.

\section{Conclusions}

The effect of strain rate on the in-plane compressive response of fibre composite with toughened matrix system is studied using a series of quasi-static $\left(\approx 1 \mathrm{e}-3 \mathrm{~s}^{-1}\right)$ and high rate $\left(\approx 200 \mathrm{~s}^{-1}\right)$ experiments using off-axis coupons. It is concluded that strain rate has significant effect on the compression-in-plane shear response of FRPs leading to an average increase of $43 \%$ in the failure strength, while measured axial modulus is relatively unaffected by strain rate. Puck failure theory provides reasonable predictions of the experimentally observed failure envelopes at both quasi-static and high rates. Further exploration of the effect of strain rate on the longitudinal shear and transverse tensile behaviour of FRPs is left for a future study.

The authors would like to acknowledge the financial support of RollsRoyce plc and Innovate UK. The authors are grateful to Rolls-Royce plc for giving permission to publish this work.

\section{References}

1. H. Koerber, J. Xavier, P.P. Camanho, Mech. Mater. 42, 1004-1019 (2010)

2. K. Naresh, K. Shankar, B.S. Rao, R. Velmurugan, Composites Part B 100, 125-135 (2016)

3. Y. Tao, H. Chen, K. Yao, H. Lei, Y. Pei, D. Fang, Int J Solids Struct. 113-114, 37-46 (2017)

4. J.D. Schaefer, B.T. Werner, I.M. Daniel, Exp. Mech. 54, 111-1120 (2014) 
Table 1. Effect of strain rate on axial modulus and failure strength under compression.

\begin{tabular}{|c|c|c|c|c|c|c|}
\hline & \multicolumn{3}{|c|}{ Axial Modulus [GPa] } & \multicolumn{3}{|c|}{ Normalized Strength [MPa] } \\
\hline $\begin{array}{c}\text { Specimen } \\
\text { Orientation }\end{array}$ & QS & HR & $\begin{array}{c}\text { Change } \\
\%\end{array}$ & QS & HR & $\begin{array}{c}\text { Change } \\
\%\end{array}$ \\
\hline $30^{\circ}$ & 22.37 & 25.40 & $\mathbf{1 3 . 5 4}$ & 1.06 & 1.43 & 34.90 \\
$45^{\circ}$ & 14.70 & 14.75 & 0.34 & 0.92 & 1.36 & 47.82 \\
$60^{\circ}$ & 11.45 & 11.96 & 4.45 & 0.98 & 1.41 & 43.87 \\
$90^{\circ}$ & 9.53 & 9.50 & -0.30 & 0.95 & 1.41 & $\mathbf{4 8 . 4 2}$ \\
\hline
\end{tabular}

5. H. Kolsky, Proc. Phys. Soc. London, Sect. B. 62, 676$700(1949)$

6. G.T. Gray III , ASM Handbook, 8, 462-476 (2000)
7. A, Puck, H.,Schürmann, Compos. Sci. Technol. 58, 1045-1067 (1998) 